\title{
FATORES QUE INFLUENCIAM A VARIAÇÃO DA MASSA LINEAR NA PRODUÇÃO DE ARAMES CA60*
}

Enrico Tosolini ${ }^{1}$

\section{Resumo}

O controle da massa linear dos arames CA60 vem sendo tratado com máxima prioridade, devido ao constante esforço da indústria nacional de reduzir os custos e melhorar a eficiência de produção. O trabalho é um estudo teórico dos fatores físicos que influenciam na variação da massa linear. Para desenvolver a análise, o problema foi simplificado para reconduzi-lo a um problema linear de fácil análise e com parâmetros intuitivos para fácil aplicação dos conceitos nos problemas diários da produção.

Palavras-chave: Laminação a frio; Variação massa linear; Arames CA60; Variação do fio-máquina; Rigidez conjuntos de laminação; Lubrificantes na laminação a frio; Temperaturas de funcionamento; Dilatação arame; Dilatação anéis.

\section{FACTORS AFFECTING DE VARIATION OF THE LINEAR MASS IN THE PRODUCTION OF COLD ROLLED REBAR WIRES}

\section{Abstract}

The control of the linear mass has become a relevant issue, due to the constant aim to reduce costs and improve the efficiency of production of rebar products. The study makes a theoretic approach to the physical factors which influence the variation of the linear mass. To develope the analysis, the problem has been reduced to a simplified linear problem, whose parameters are well intuitive and easy applying on the everyday production situations.

Keywords: Cold rolling; Linear mass variation; CA 60 rebar wire; Wire rod variation; Rigidity of lamination equipment; Cold roll wire lubrication; Working temperatures; Wire dilatation; Lamination rolls dilatation. 


\section{INTRODUÇÃO}

Cada vez mais o assunto do controle da massa linear vem tendo importância no industrial de arames. Os arames laminados a frio do tipo CA 60, pela norma ABNT NBR 7480 , admitem uma tolerância de até $6 \%$, pra mais ou pra menos, da massa linear em respeito à bitola gravada no arame. A possibilidade de ter uma produção estável para ficar nos limites inferiores da norma sem sair dos parâmetros estabelecidos, pode proporcionar, para o produtor que tome estes cuidados, um importante diferencial na renda do produto final. Considerando um custo por quilo do fio maquina de aproximadamente $2 \mathrm{R} \$$, e uma produção mensal de aproximadamente 2.000 toneladas (uma maquina em três turnos), dá para entender como uma poupança de $3 \%$ no peso do produto utilizado, represente um ganho de $\mathrm{R} \$ 120.000$.

Os objetivos deste trabalho são de fornecer uma base teórica, além de uma explicação prática, dos parâmetros que influenciam a variação da massa linear e dos cuidados a serem tomados para estabilizar a própria produção de laminados.

Como há uma escassez de literatura a respeito da laminação a frio de arames, foram utilizadas fórmulas emprestadas da trefilação, adaptadas para o processo da laminação à frio.

\section{MATERIAIS E MÉTODOS}

Neste estudo foram considerados os seguintes fatores que influenciam a variação das bitolas durante a produção:

1. Variação do Fio-Maquina.

2. Rigidez do Conjunto de Laminação e resposta à variações dimensionais do material.

3. Lubrificação do arame.

4. Temperatura do arame.

5. Temperatura das ferramentas em contato com arame (anéis laminadores e suportes).

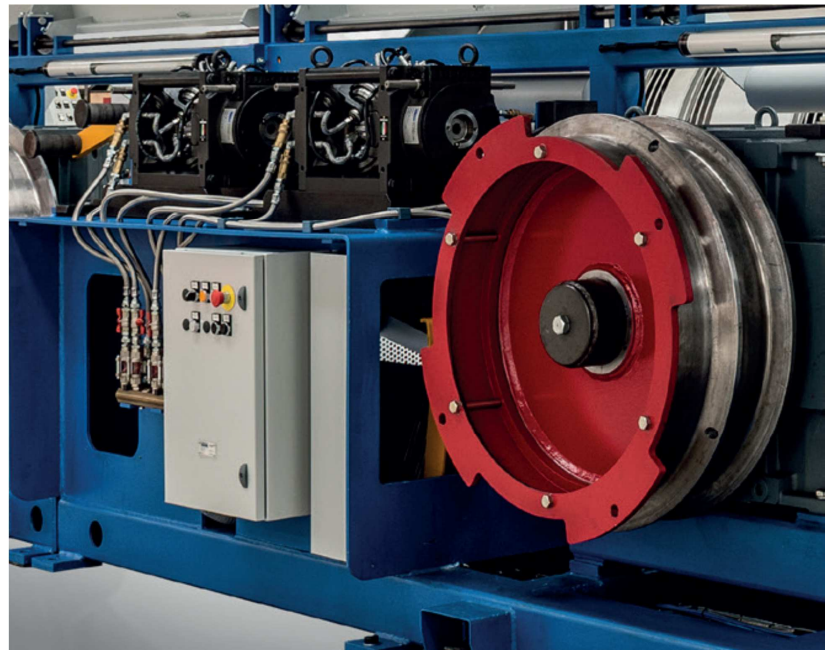

Figura 1. Bloco de laminação para CA60.

Cada um dos fatores elencados acima foi analisado de forma isolada e independente de outros fatores.

Não foram considerados os efeitos da combinação dos fatores acima mencionados. 
Segue um resumo dos parâmetros físicos utilizados para as simulações.

Tabela 1. Parâmetros físicos

\begin{tabular}{|c|c|c|c|c|}
\hline ELEMENTO & PAR. & DESCRICAO & UM & VALOR \\
\hline Material & - & Fio Máquina SAE 1006 - Te 350 Mpa & - & - \\
\hline Material & $\rho_{a}$ & Peso Específico do Aço do Fio-Máquina & {$[\mathrm{g} / \mathrm{cm} 3]$} & 7,85 \\
\hline Material & $\alpha_{a}$ & Coeficiente Dilataçao Linear Aço & {$\left[{ }^{\circ} \mathrm{C}^{\wedge}-1\right]$} & $11,0 \mathrm{E}-06$ \\
\hline Fio-Máquina & $A$ & Amplitude da variação do diâmetro $(-+)$ & {$[\mathrm{mm}]$} & 0,10 \\
\hline Fio-Máquina & $P$ & Periodo da variação & {$[\mathrm{m}]$} & 43,00 \\
\hline Cassete & $K_{c}$ & Rigidez do Conjunto de Laminação & {$[\mathrm{N} / \mathrm{mm}]$} & $3,00 \mathrm{E}+04$ \\
\hline Lubrificante & $\mu_{l}$ & $\begin{array}{c}\text { Coeficiente de fricção lubrificado HM-Aço } \\
\text { (lubrificado) }\end{array}$ & {$[-]$} & 0,05 \\
\hline Lubrificante & $\mu_{d}$ & $\begin{array}{c}\text { Coeficiente de fricção lubrificado HM-Aço } \\
\text { (seco) }\end{array}$ & {$[-]$} & 0,15 \\
\hline Lubrificante & $t_{l}$ & $\begin{array}{c}\text { Tempo de zeramento das propriedades } \\
\text { lubrificantes }\end{array}$ & {$[\mathrm{s}]$} & $18.000,00$ \\
\hline Lubrificante & $m_{l}$ & $\begin{array}{c}\text { Coeficiente de aumento do coeficiente de } \\
\text { fricção }\end{array}$ & {$[1 / \mathrm{s}]$} & $-5,56 \mathrm{E}-06$ \\
\hline Produção & $P_{a}$ & Peso da bobina de CA60 final & {$[\mathrm{kg}]$} & $2.500,00$ \\
\hline Produção & $t_{p a}$ & Tempo para produção de Pa & {$[\mathrm{min}]$} & 21,50 \\
\hline Produção & $t_{c b}$ & Tempo para troca da bobina de Pa & {$[\mathrm{min}]$} & 3,50 \\
\hline Produção & $t_{t c}$ & Tempo Total do ciclo & {$[\mathrm{min}]$} & 25,00 \\
\hline Rolete & $\alpha_{w}$ & Coeficiente Dilatação Linear Carbeto T. & {$\left[{ }^{\circ} \mathrm{C}^{\wedge}-1\right]$} & $6,0 \mathrm{E}-06$ \\
\hline
\end{tabular}

\subsection{Variação do Fio-Máquina}

Conforme indicado nos catálogos dos maiores produtores de aço, foi considerada uma tolerância dimensional $\pm 0,1 \mathrm{~mm}$ para o fio-máquina.

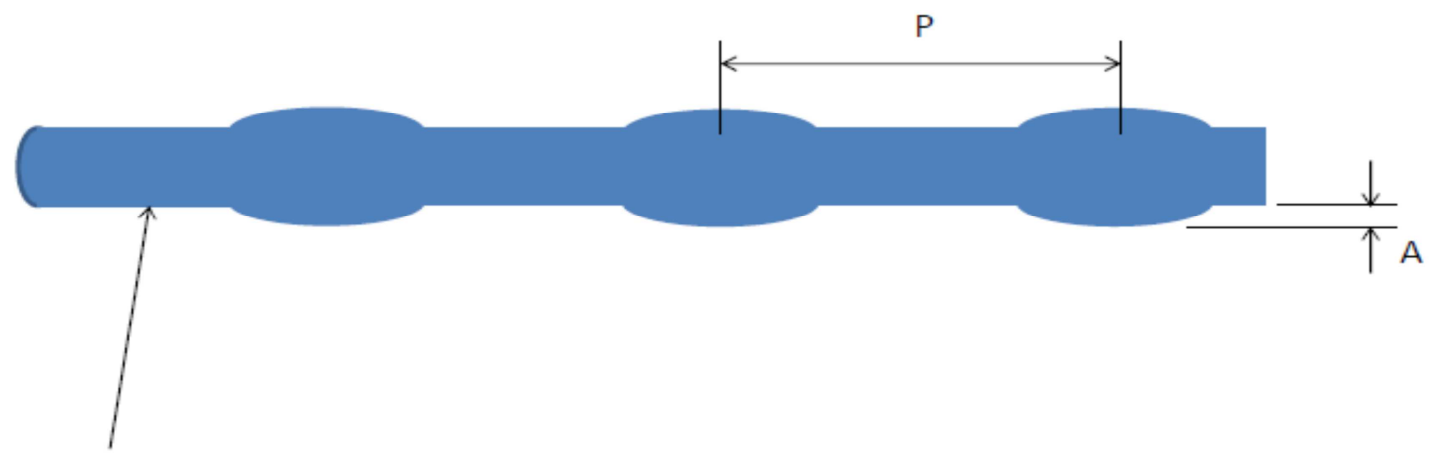

ARAME

Figura 2. Fio-Máquina.

Foi considerada uma variação sinusoidal do diâmetro do fio-máquina com período da variação $P$ e Amplitude da variação do diâmetro (-+) $A$ conforme a Tabela $1 \mathrm{e}$ velocidade de entrada no primeiro bloco $V_{01}=7,5 \mathrm{~m} / \mathrm{s}$.

Segue a fórmula utilizada:

$$
D_{0}(t)=D_{0 n}+A \sin (\omega t)
$$


Na fórmula entendem-se:

$D_{0}(t)$ : Diâmetro de entrada no Cassete em função do tempo

$D_{0 n}$ : Diâmetro Nominal Fio-Máquina

$$
\begin{aligned}
& \omega=\frac{2 \pi}{T} \\
& T=\frac{P}{V_{01}}
\end{aligned}
$$

A variação da bitola do Fio-Máquina impacta diretamente nas forças de laminação e é contrastada pela rigidez do Conjunto de Laminação, conforme tratado no paragrafo seguinte.

\subsection{Rigidez do Conjunto de Laminação e resposta à variações do Fio-Maquina}

Entende-se como Conjunto de Laminação o conjunto formado por todos os componentes responsáveis para laminação, ou seja:

- Anéis de Laminação/Rolete

- Suportes/mancais/Rolamentos

- Pino do Suporte

- Corpo do Cassete de Laminação.

Todos estes componentes, montados com as respectivas folgas e constantes elásticas, formam um conjunto elástico que será considerado como único para fins da nossa análise.
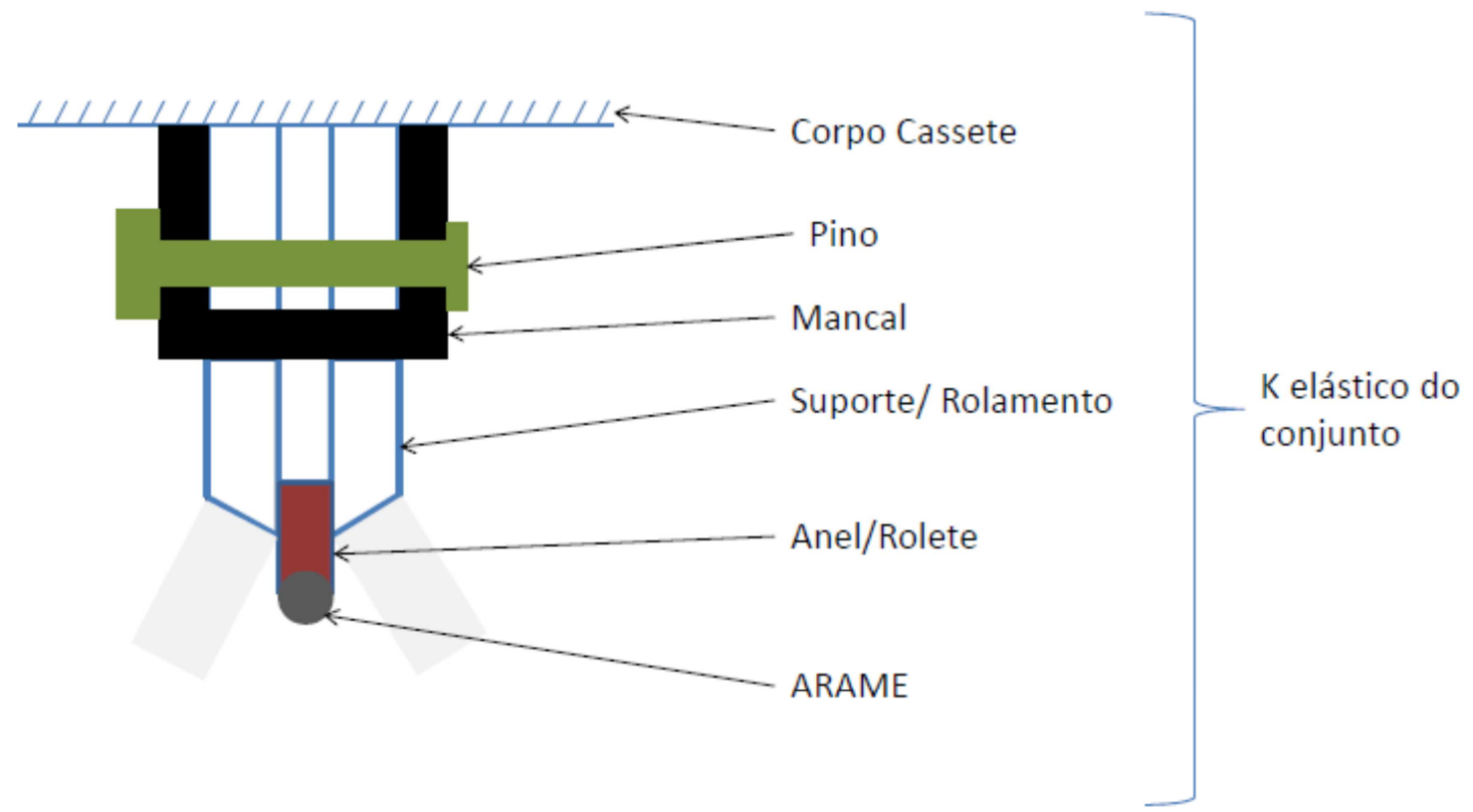

Figura 3. Conjunto de Laminação.

Para esse escopo foi considerado $K_{c}$ um global que pudesse levar em conta todos os comportamentos elásticos de reação às forças de laminação, conforme Fig. 3. Foi considerado que a qualquer variação de bitola do Fio-Máquina, o Conjunto de Laminação responda com uma reação proporcional à própria constante elástica $K_{c}$. A convenção utilizada para as forças e deslocamentos é conforme a Fig. 4.

A relação entre uma força derivada da variação dimensional no Fio-Máquina e os deslocamentos/Constante $K_{c}$, numa condição de equilíbrio instantâneo é a seguinte: 


$$
\begin{gathered}
F_{A}=F_{l}=K_{c} \Delta x \\
\Delta x=\frac{F_{A}}{K_{c}}
\end{gathered}
$$

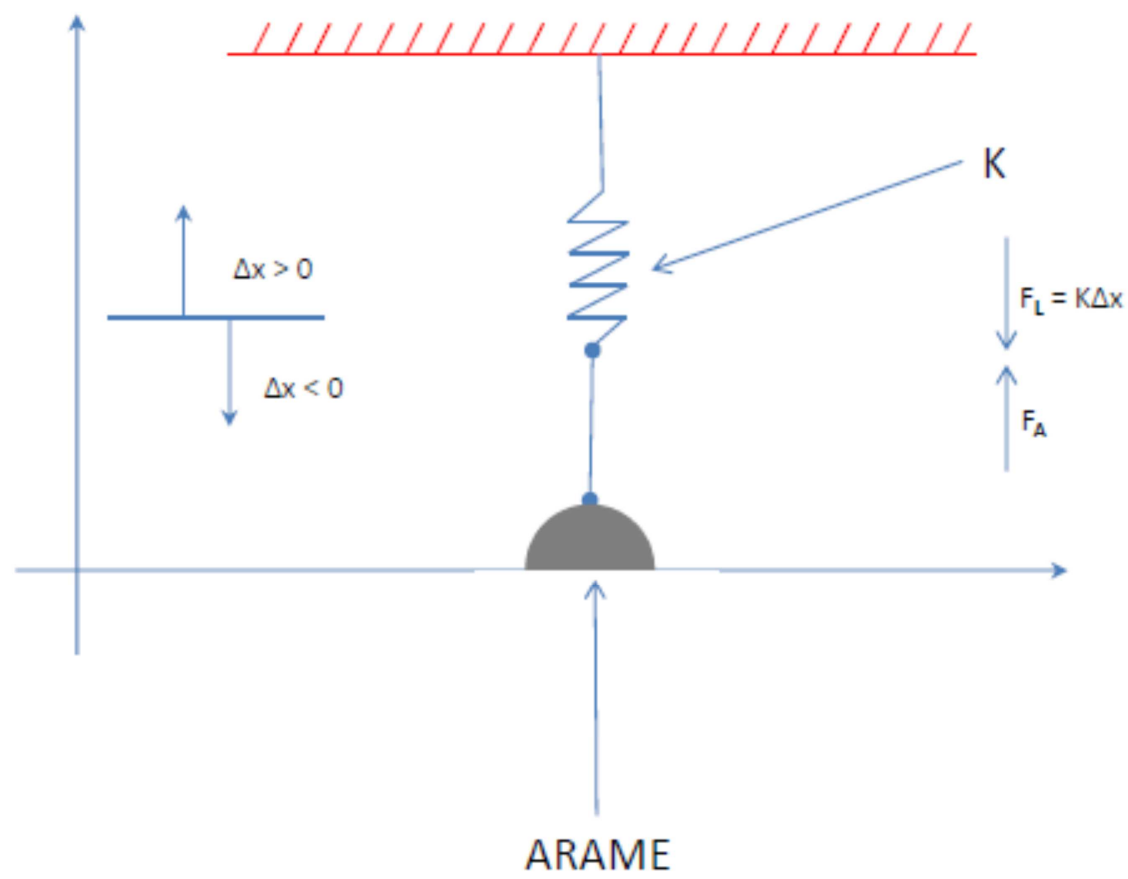

Figura 4. Rigidez do Conjunto de Laminação.

A Força de Laminação instantânea bem como as pressões radiais e longitudinais, foram calculadas utilizando os parâmetros geométricos da secção de laminação e a fórmula de Siebel, ajustada com parâmetros aptos para laminação:

$$
F_{z(t)}=A_{1 n} \cdot R_{e m n} \cdot\left(\ln \frac{A_{o}(t)}{A_{1 n}}+\frac{2 \alpha_{l}(t)}{3}+\frac{\mu_{l}(t)}{\alpha_{l}(t)} \cdot \ln \frac{A_{0}(t)}{A_{1 n}}\right)
$$

Onde os termos são os seguintes:

$F_{z(t)}$ : Força de Tração Axial calculada ao variar no tempo.

$A_{1 n}:$ Área de saída Nominal.

$R_{e m n}:$ Média das tensões de escoamento antes e depois da redução.

$A_{o}(t)$ : Área de entrada do material na redução, calculada usando o diâmetro $D_{0}(t)$ da (1).

$\alpha_{l}(t)$ : semiângulo efetivo para laminação calculado instantaneamente conforme segue:

\section{Sendo:}

$$
\alpha_{l}(t)=\arctan \left(\frac{D_{0}(t)-D_{1 n}}{\sqrt{4 R\left(D_{0}(t)-D_{1 n}\right)-\left(D_{0}(t)-D_{1 n}\right)^{2}}}\right)
$$

R: o Raio externo do anel.

$D_{1 n}:$ O diâmetro nominal de saída do arame.

$\mu_{i l}(t)=\Phi(t) \cdot \mu_{l}$ : coeficiente ideal de fricção corrigido para laminação e calculado Instantaneamente.

$\Phi=1+0,12 \cdot(\Delta(t)-1):$ o Fator de não homogeneidade de Wistreich.

$\mu l$ : o coeficiente de atrito conforme a Tabela 1.

$\Delta(t)$ o comprimento de secção de laminação em função do tempo. 
A variação das forças de laminação é importante sendo que elas interagem diretamente com as forças elásticas do Conjunto de Laminação.

$O$ deslocamento decorrente desta açao foi chamado de $\Delta D(t)_{f m}$, entendendo que há um aumento do diametro do arame igual ao cedimento do Conjunto de Laminação.

\subsection{Sabão Lubrificante}

O sabão lubrificante sofre um desgaste natural do poder de lubrificação, devido a contaminação de fatores externo (umidade, sujeira, carepa).

Foi então explicitada uma função linear para levar em conta a perda do poder de lubrificação conforme segue:

Sendo

$$
\mu_{l}(t)=\mu_{l}+X_{l u b} \cdot t
$$

$$
X_{l u b}=\frac{\left(\mu_{d}-\mu_{l}\right)}{t_{c}}
$$

Onde

$\mu_{d}$ : é o coeficiente de fricção a seco.

$t_{c}$ : é o tempo de desgaste completo das propriedades do sabão lubrificante.

Substituindo a (8) na equação da força de tração (6) e nas demais equações envolvidas, è possível monitorar a influência da variação do coeficiente de fricção na variação das pressões de contato.

O deslocamento decorrente desta açao foi chamado de $\Delta D(t)_{l}$ entendendo que há um aumento do diametro do arame igual ao cedimento do Conjunto de Laminação.

\subsection{Temperatura do Arame}

A variação da temperatura do arame proporciona um aumento ou diminuição do diâmetro do mesmo. Como consequência, o efeito da variação da temperatura pode ser assimilado ao processo de variação de bitola do Fio-Máquina e tratado do mesmo jeito, ou seja, considerando as suas interações com o sistema elástico do Conjunto de Laminação. A variação ocorre principalmente nas fases transitórias do processo (começo e término da bobina) e pode ser representada pela fórmula da variação no campo elástico:

$$
\Delta D_{t f}(t)=D_{1 n} \cdot \alpha_{a} \cdot\left(T_{a}(t)-T_{r a}\right)
$$

Sendo:

$\alpha_{a}$ : O coeficiente de dilatação linear do aço conforme Tabela 1.

$T_{a}(t)$ : a temperatura do arame em cada instante.

$T_{r a}$ : temperatura de referência do arame (considerada como a temperatura do regime estacionário, ou seja, quando acontece a produção da maior quantidade de produto durante o ciclo de produção).

\subsection{Temperatura dos Roletes}

Também a variação da temperatura dos roletes proporciona um aumento/diminuição do diâmetro do arame, como consequência da contração/dilatação dos anéis durante as fases transitórias. Diferentemente da variação de temperatura no arame, a variação nos roletes acontece na parte do Conjunto de Laminação e por isso é levada em conta como pura variação geométrica e sem considerar as interações desta variação com o sistema elástico do Conjunto de Laminação. Sendo a rigidez 
do carbeto de tungstênio muito maior do que a do aço considera-se que a variação seja transferida toda para o arame como variação de diâmetro do arame mesmo. Então, a variação do diâmetro do arame em função da variação do diâmetro dos roletes no campo elástico pode ser considerada como:

Sendo:

$$
\Delta D_{t r}(t)=2 \cdot R \cdot \alpha_{w} \cdot\left(T_{w}(t)-T_{r w}\right)
$$

$\alpha_{w}$ : O coeficiente de dilataçao linear do carbeto de tungstenio conforme Tabela 1.

$T_{w}(t)$ : a temperatura do rolete em cada instante.

$T_{r w}$ : temperatura de referencia do rolete (considerada como a temperatura durante a fase a regime estacionário do ciclo de produção ou seja, quando acontece a produção da maior quantidade de produto).

\subsection{Definiçao da Massa Linear e Soma dos Efeitos de Variação}

A variação da massa linear fica entao definida como:

Onde:

$$
\Delta M L(t)=\frac{D_{1}(t)^{2}}{D_{1 n^{2}}}-1
$$

$D_{1}(t)$ : Diâmetro Real de saída do arame no tempo.

$D_{1 n}$ : Diâmetro Nominal de saída do arame.

Sendo assim o Diâmetro Real pode ser também expresso como:

$$
D_{1}(t)=D_{1 n}+\Delta D(t)_{f m}+\Delta D(t)_{l}+\Delta D_{t f}(t)+\Delta D_{t r}(t)
$$

A união das formulas (12) e (13), então, leva em conta a variação da massa linear em função dos fatores que influenciam a mesma.

\section{RESULTADOS E DISCUSSÃO}

A fim de simular a variação da Massa Linear em um ciclo com regime misto estacionário-transitório, foi estudada a laminação de um arame bitola $4,20 \mathrm{~mm}$, produzido em uma maquina laminadora de três passes a partir de Fio-maquina $\varnothing 6,50 \mathrm{~mm}$. Foi considerada a produção de uma bobina de $2.500 \mathrm{~kg}$ em um tempo de 25 minutos, sendo 21,5 minutos de laminação e 3,5 minutos para troca do carretel.

A rampa de aceleração/desaceleração foi considerada de 20 segundos.

O tempo de resfriamento e de reaquecimento completo foi considerado igual entre eles e ao tempo de troca da bobina (3,5 minutos, ou seja, 210 segundos).

Os parâmetros de temperatura em regime estacionário foram considerados conforme Tabela 2, enquanto os valores mínimos alcançados durante a parada da máquina para troca do carretel foram resumidos na Tabela 3.

Tabela 2. Temperaturas no regime estacionário

\begin{tabular}{|r|r|r|r|r|r|r|r|}
\hline $\begin{array}{c}\text { Draft } \\
{[-]}\end{array}$ & $\begin{array}{c}\text { Inlet } \\
\text { Diameter } \\
{[\mathbf{m m}]}\end{array}$ & $\begin{array}{c}\text { Outlet } \\
\text { Diameter } \\
{[\mathbf{m m}]}\end{array}$ & $\begin{array}{c}\text { Outlet } \\
\text { Speed } \\
{[\mathbf{m} / \mathbf{s}]}\end{array}$ & $\begin{array}{c}\text { Wire } \\
\text { Temp. } \\
{\left[\mathbf{C}^{\circ}\right]}\end{array}$ & $\begin{array}{c}\text { Roll } \\
\text { Holder } \\
\text { Temp. } \\
{\left[\mathbf{C}^{\circ}\right]}\end{array}$ & $\begin{array}{c}\text { Ring } \\
\text { Temp. } \\
{\left[\mathbf{C}^{\circ}\right]}\end{array}$ & $\begin{array}{c}\text { Pin } \\
\text { Temp. } \\
{\left[\mathbf{C}^{\circ}\right]}\end{array}$ \\
\hline $\mathbf{1}$ & $\mathbf{6 , 5 0 0}$ & $\mathbf{5 , 5 0 0}$ & 10,5 & 112,4 & 100,0 & 80,0 & 28,0 \\
\hline $\mathbf{2}$ & $\mathbf{5 , 5 0 0}$ & $\mathbf{4 , 7 5 0}$ & 14,1 & 104,2 & 110,0 & 90,0 & 29,0 \\
\hline $\mathbf{3}$ & $\mathbf{4 , 7 5 0}$ & $\mathbf{4 , 2 0 0}$ & 18,0 & 99,9 & 120,0 & 100,0 & 30,0 \\
\hline
\end{tabular}


Tabela 3. Temperaturas minimas

\begin{tabular}{|r|r|r|r|r|r|r|r|}
\hline $\begin{array}{c}\text { Draft } \\
{[-]}\end{array}$ & $\begin{array}{c}\text { Inlet } \\
\text { Diameter } \\
{[\mathbf{m m}]}\end{array}$ & $\begin{array}{c}\text { Outlet } \\
\text { Diameter } \\
{[\mathbf{m m}]}\end{array}$ & $\begin{array}{c}\text { Outlet } \\
\text { Speed } \\
{[\mathbf{m} / \mathbf{s}]}\end{array}$ & $\begin{array}{c}\text { Wire } \\
\text { Temp. } \\
{\left[\mathbf{C}^{\circ}\right]}\end{array}$ & $\begin{array}{c}\text { Roll } \\
\text { Holder } \\
\text { Temp. } \\
{\left[\mathbf{C}^{\circ}\right]}\end{array}$ & $\begin{array}{c}\text { Ring } \\
\text { Temp. } \\
{\left[\mathbf{C}^{\circ}\right]}\end{array}$ & $\begin{array}{c}\text { Pin } \\
\text { Temp. } \\
{\left[\mathbf{C}^{\circ}\right]}\end{array}$ \\
\hline $\mathbf{1}$ & $\mathbf{6 , 5 0 0}$ & $\mathbf{5 , 5 0 0}$ & 0,0 & 101,2 & 90,0 & 72,0 & 25,2 \\
\hline $\mathbf{2}$ & $\mathbf{5 , 5 0 0}$ & $\mathbf{4 , 7 5 0}$ & 0,0 & 93,8 & 99,0 & 81,0 & 26,1 \\
\hline $\mathbf{3}$ & $\mathbf{4 , 7 5 0}$ & $\mathbf{4 , 2 0 0}$ & 0,0 & 89,9 & 108,0 & 90,0 & 27,0 \\
\hline
\end{tabular}

A variação de velocidade e temperatura, durante o regime transitório, foi considerada linear.

Plotando em um gráfico os resultados obtidos para $\Delta \boldsymbol{M L}$ (Figura 5), dá para destacar:

- A influência da variação da temperatura no regime transitório entre 0-210 seg e entre 1290-1500 seg.

- A influência da variação do fio-máquina com andamento senuzoidal. Ela é mais destacada no primeiro passo e vai diminuindo nos dois passos seguintes.

- A influência da diminuição do poder de lubrificação, percebida no aumento gradual da massa linear é destacada melhor na parte de funcionamento a regime estacionário (parte central).

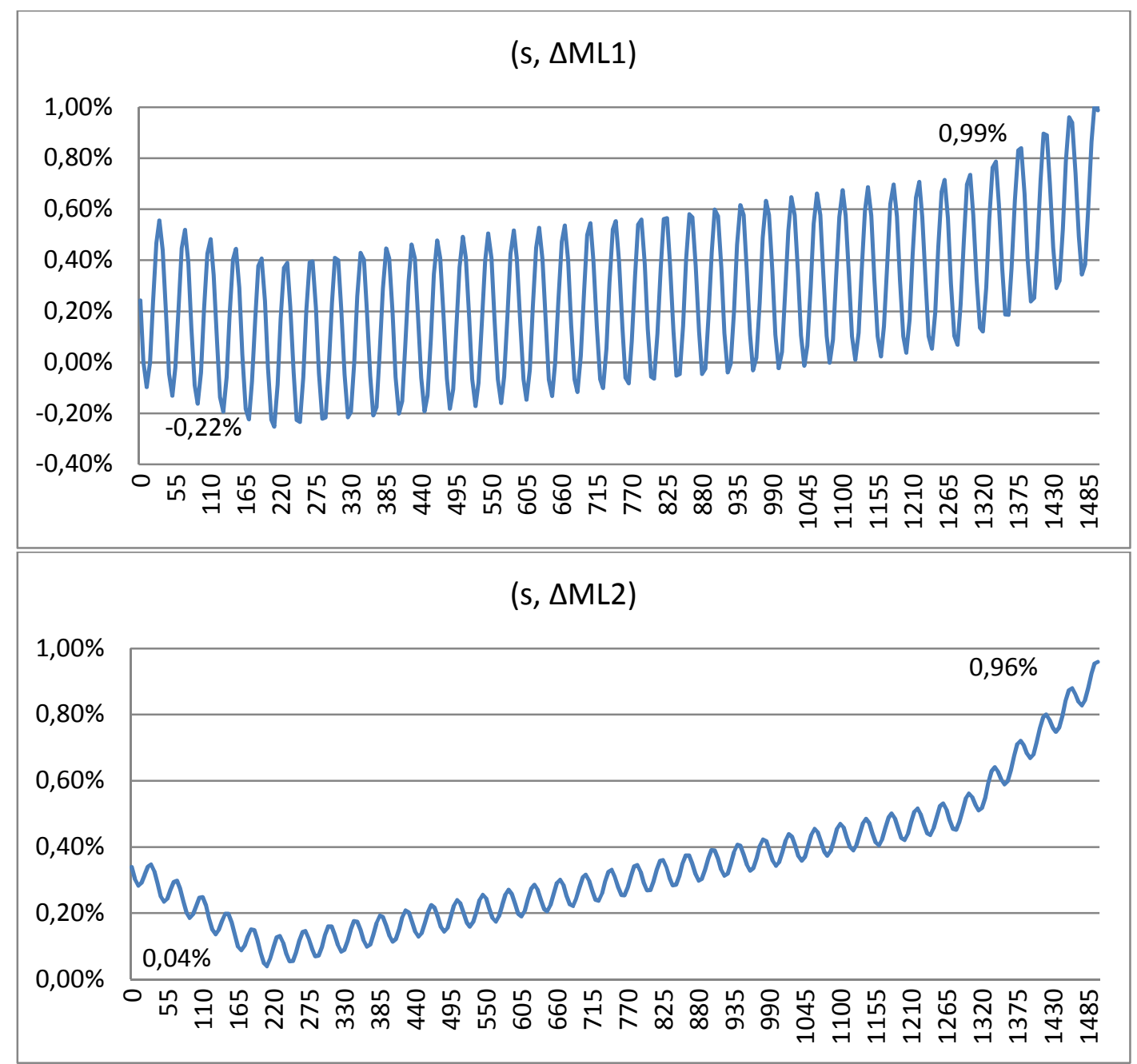


$(\mathrm{s}, \Delta \mathrm{ML3})$

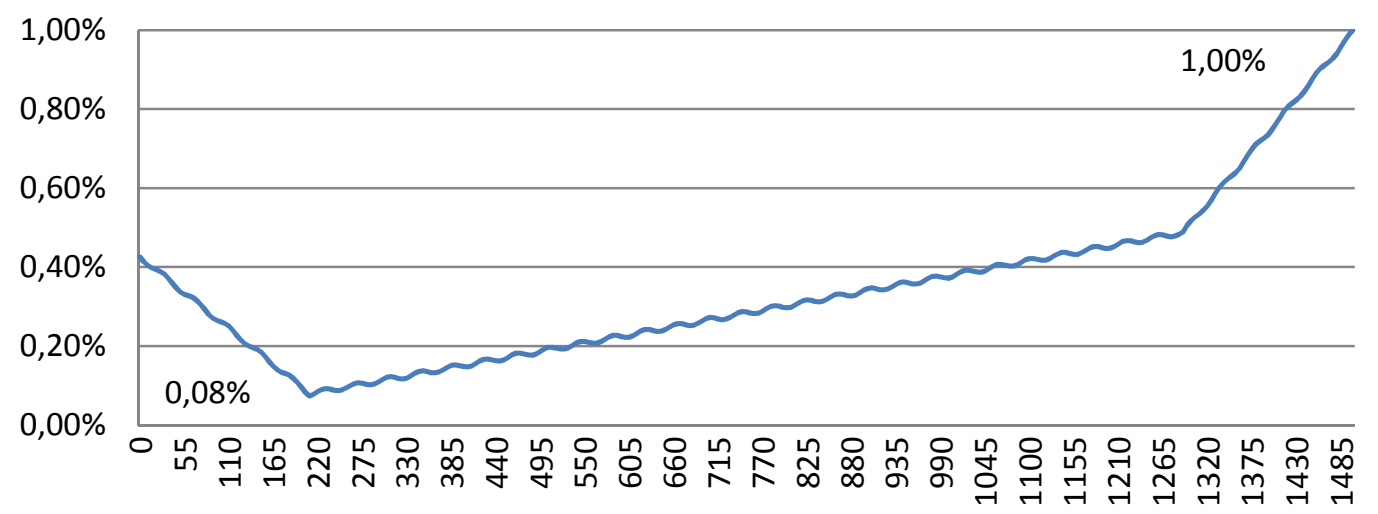

Figura 5. Variação da Massa Linear

Para avaliar a dependência da $\Delta \boldsymbol{M} \boldsymbol{L}$ da Rigidez do Conjunto de Laminação, da temperatura e do lubrificante, é possível variar esses parâmetros e perceber:

- Uma variação da amplitude das oscilações proporcional com $\boldsymbol{K}_{\boldsymbol{c}}$. Uma variação da $\Delta \boldsymbol{M L}$ no transitório proporcional com a diferença entre temperatura a regime estacionário e temperatura mínima.

- Uma variação da pendência no trecho em regime estacionário inversamente proporcional com $\boldsymbol{m}_{\boldsymbol{l}}$, ou seja com Coeficiente de aumento do coeficiente de fricção.

\section{CONCLUSÕES}

O modelo elaborado dá para trazer as seguintes conclusões:

- Uma rigidez maior do Conjunto de Laminação, entendido como conjunto de roletes, suportes porta-rolo, estrutura do cassete, leva a uma maior estabilidade em geral do processo de produção e a uma diminuição das variações RELATIVAS da massa linear.

- Um controle melhor das temperaturas nos transitórios leva a uma diminuição importante das variações ABSOLUTAS da massa linear.

- Um melhor resfriamento do Conjunto de Laminação, que geralmente tem efeitos muito benéficos em relaçao a outros fatores relacionados à durabilidade do ferramental, nao tem um impacto muito grande no controle da massa linear, a não ser de manter baixa a TEMPERATURA MÁXIMA no período de regime constante, então de diminuir a variação absoluta dos valores de resfriamento dos conjuntos. Seria muito mais eficaz controlar as TEMPERATURAS MÍNIMAS na fase do regime transitório.

- Um melhor controle e cuidado com a lubrificação leva a uma maior estabilidade TENDENCIAL da massa linear.

- O modelo proposto, mesmo que simplificado, é um modelo versátil, sendo que considera a variação da massa linear como soma de fatores relacionados a forças-rigidez de um lado e temperatura do outro. A inclusão do estudo de demais fatores que impactam no processo de laminação, poderá ser feita desde que os relacionando às forças ou as temperaturas. 


\section{Agradecimentos}

Marco Castenetto - Danieli Morgardshammar Bar \& Wirerod Mills. Clément Barrat - Condat do Brasil - Divisão Arames e Tubos.

\section{REFERÊNCIAS}

1 Enghag P. Steel Wire Technology Edição 4 Örebro - Sweden; Repro Örebro University; 2009

2 Bitkov V. Expediency of Roller Dies Application in Wire Drawing Part1 - Wire \& Cable Technology International / Janeiro 2008 Part2 - Wire \& Cable Technology International / Março 2008.

3 Basuc D. Cenni sui processi di Lavorazione per deformazione. Disponível em: http://corsiadistanza.polito.it/corsi/pdf/9638A/sez1.pdf

4 Beretta S. Appunti di Costruzione di Macchine 2. Disponível em: http://cm2.mecc.polimi.it/Lezioni/CM2_19.pdf

5 Broggiato Solidi assialsimmetrici - Dischi. Disponível em: http://dma.dma.uniroma1.it/users/broggiato/cdm/roma/vo/ecdm/dispense/[26]Dis chi.pdf

6 Esposito L. Stato di sforzo in solidi assialsimmetrici. Disponível em: http://www.cdmunicas.it/files/2010_CDM_Solidiassialsimmetrici.pdf

7 Petrucci G. Lezioni di Costruzioni di Macchine.

Disponível em: http://www.unipa.it/giovanni.petrucci/Disp/Dischi\%20rotanti.pdf 